\title{
Fechamento de diastema e reanatomização com cerâmica odontológica: relato de caso
}

Diastema closure and rehabilitation with dental ceramics: case report

Cierre de diastema y reanatomización con cerámica odontológica: relato de caso

\author{
Lia Beatrice Sampaio ANDRADE \\ Cristiano Pires e SILVA
}

Sybilla Torres DIAS

Departamento de Clínica e Prótese, Policlínica Odontológica, UEA Univ. do Estado do Amazonas, 69065-132, Manaus - AM, Brasil

\begin{abstract}
Resumo
Uma das problemáticas da ortodontia é finalizar o tratamento com a presença de diastemas, no entanto, é possível solucionar de várias maneiras, uma delas é a confecção de restaurações indiretas, como os laminados cerâmicos. O crescente desenvolvimento tecnológico dos materiais odontológicos reabilitadores, em especial os cimentos e sistemas adesivos, vem melhorando adesividade ao esmalte/dentina fazendo união química e mecânica e aumentandotambém a resistência estrutural, conferindo características mais estéticas, duradouras e confiáveis dessas restaurações. O presente trabalho relata um caso clínico de fechamento de diastemas múltiplos, pós tratamento ortodôntico, utilizando lentes de contato dental em dissilicato de lítio. O resultado foi satisfatório esteticamente além de solucionar os problemas como: fechamentodos diastemas resultantes da ortodontia, pontos de contatos, fonética e satisfação pessoal do paciente.
\end{abstract}

Descritores: Diastema; Estética Dentária; Facetas Dentárias.

\section{Abstract}

One of the problems of orthodontics is to finish the treatment with the presence of diastema, however, it is possible to solve in several ways, one of them is the making of indirect restorations, such as ceramic laminates. The increasing technological development of rehabilitating dental materials, especially cements and adhesive systems, has been improving adhesion to the enamel / dentin making chemical and mechanical bonding and also increasing the structural resistance, giving more aesthetic, durable and reliable characteristics of these restorations. This paper reports a clinical case of multiple diastema closure after orthodontic treatment using lithium disilicate dental contact lenses. The result was aesthetically satisfactory as well as solving problems such as: closing the diastema resulting from orthodontics, contact points, phonetics and personal satisfaction of the patient.

Descriptors: Diastema; Esthetics, Dental; Dental Veneers.

\section{Resumen}

Una de las problemáticas de la ortodoncia es finalizar el tratamiento con la presencia de díastemas, sin embargo, es posible solucionar de varias maneras, una de ellas es la confección de restauraciones indirectas, como los laminados cerámicos. El creciente desarrollo tecnológico de los materiales odontológicos rehabilitadores, en especial los cementos y sistemas adhesivos, viene mejorando adhesividad al esmalte / dentina haciendo unión química y mecánica y aumentando la resistencia estructural, dando características más estéticas, duraderas y confiables de esas restauraciones. El presente trabajo relata un caso clínico de cierre de díastemas múltiples, post tratamiento ortodóntico, utilizando lentes de contacto dental en disilicato de litio. El resultado fue satisfactorio esteticamente además de solucionar los problemas como: cerradodos díastemas resultantes de la ortodoncia, puntos de contactos, fonética y satisfacción personal del paciente.

Descriptores: Diastema; Estética Dental; Coronas con Frente Estético.

\section{INTRODUÇÃO}

A constante busca por um sorriso harmônico e estético eleva o nível de exigência e de expectativa dos pacientes. Esse fato propicia o desenvolvimento de novos materiais e técnicas odontológicas, visando procedimentos mais conservadores e resultados cada vez mais previsíveis esteticamente ${ }^{1}$.

Devido à evolução dos sistemas adesivos, aumentando a união restauração/dentes, é possível realizar tratamento odontológico com cerâmicas que dependendo do material utilizado, pode-se chegar bem próximo das propriedades do dente natural, proporcionando resultados estéticos, funcionais $\mathrm{e}$ com longa duração. Os tratamentos restauradores passaram a ser minimamente invasivos, com pouco desgaste dentário e sendo capaz de corrigir pequenas alterações de forma, contorno, estabilidade de cor e fechamento de diastemas ${ }^{2}$.

Os diastemas dentários são aberturas, ou espaços, entre dois dentes adjacentes na mesma arcada dentária que, muitas vezes, compromete a estética e causa insatisfação no paciente. Diferentes técnicas podem ser utilizadas para o fechamento desses, como o tratamento ortodôntico e tratamento restaurador utilizando resinas compostas ou cerâmica odontológica $^{3}$. A cerâmica odontológica apresenta-se como uma opção de material que permite manter a longevidade e a estabilidade de cor, forma e textura de restaurações, perfazendo o papel do esmalte dos elementos dentários ${ }^{4}$.

\section{CASO CLÍNICO}

Paciente F.H.F.M, 25 anos, leucoderma, sexo masculino, apresentou-se na Policlínica Odontológica da Universidade do Estado do Amazonas, através do curso de Atualização em Restaurações Cerâmicas, com a queixa principal de insatisfação com a estética do sorriso, querendo dentes mais brancos, maiores e sem espaço entre eles (Figura 1).

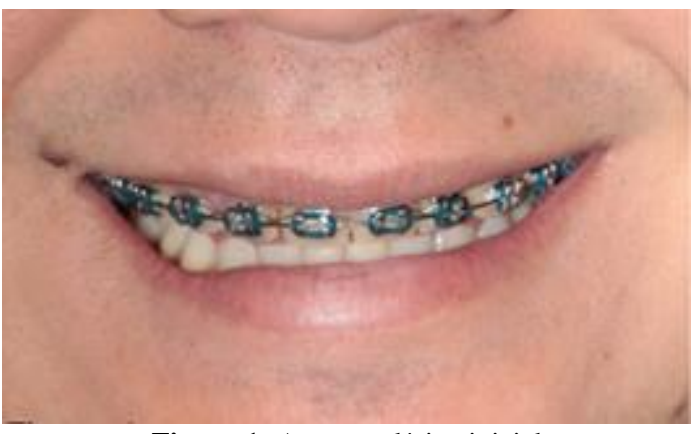

Figura 1: Aspecto clínico inicial

Paciente estava finalizando o tratamento ortodontico e ainda apresentava diastemas anteriores que o encomodavam (Figuras 2, 3 e 4). Foi solicitado uma distribuição dos diastemas entre os quatro dentes anteriores para corrigir a proporção entre os dentes. 
Este tratamento levou um mês para ser concluído.

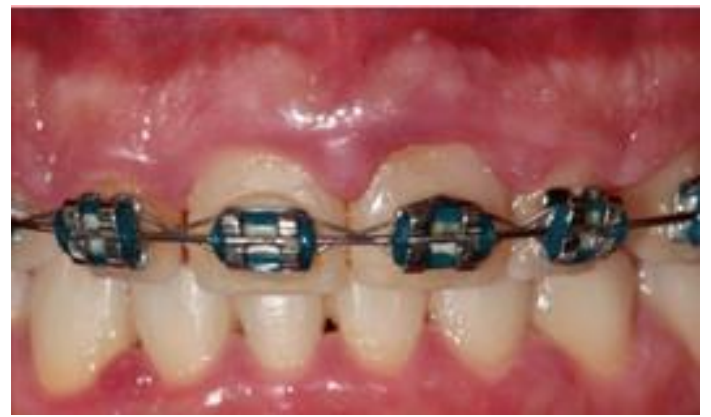

Figura 2: Aspecto intraoral mostrando crescimento gengival e coroas clinicas curtas e placas bacterianas visíveis.

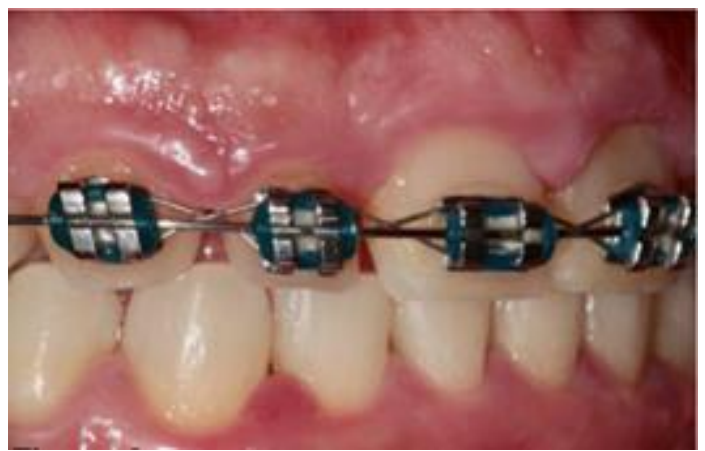

Figura 3: Vista lateral direita onde é possível ver os diastemas dos dois lados do lateral.

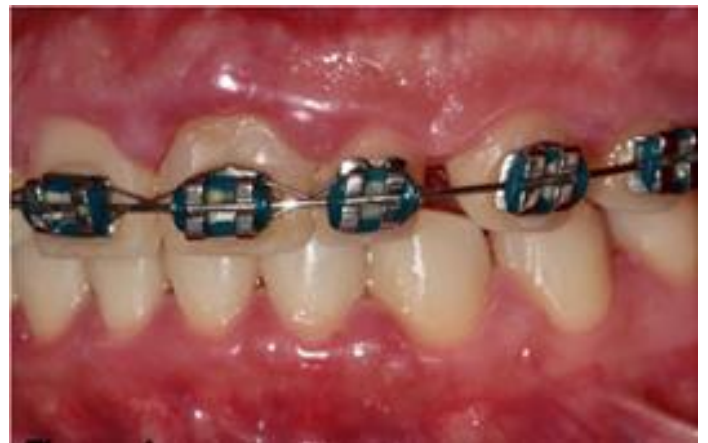

Figura 4: Vista lateral esquerda mostrando o grande diastema entre o lateral e o canino.

Após a remoção do aparelho foi realizado, pela periodontista, a gengivoplastia do elemento 13 ao 23, para remover o excesso de gengiva que recobria cerca de $2 \mathrm{~mm}$ da cervical desses dentes e correção do zênite gengival (Figuras 5 e 6). Paciente também foi orientado a melhorar o padrão de escovação, pois o mesmo apresentava higiene dental insatisfatória.

Um mês após a cirurgia o paciente retornou para realizar a moldagem anatômica, para confecção do enceramento diagnóstico e simulação das futuras restaurações em resina bisacrilica (mock-up) (Figuras 7 e 8). Após observação do mock-up em boca, foram feitas correções no enceramento na região de canino.

Em seguida foram feitos os preparos minimamente invasivos dos elementos 15 a 25 , com término em chanfrete, instrasucular e todos os angulos arredondados e polidos, em seguida foi realizado o afastamento do tecido gengival com fio \#000 Ultrapack (Ultradente) e realizado a moldagem com silicone de adição Express (3M ESPE), utilizando a técnica da dupla moldagem. As restaurações indiretas foram confeccionadas pelo laboratório Ruggeri de Maringá-PR,em dissilicato de litio pois, confere estética e resistência necessárias ao caso clínico.

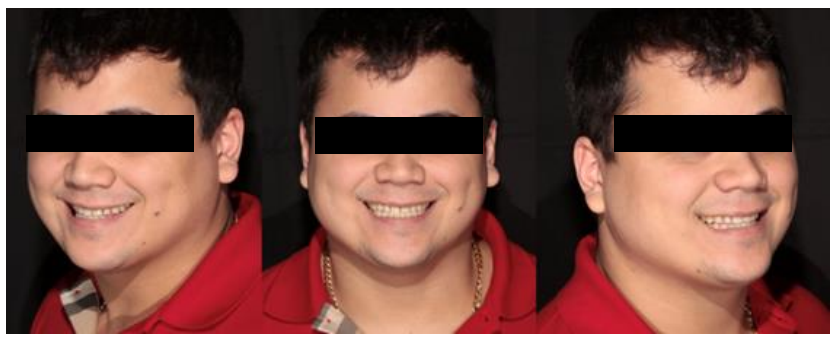

Figura 5: Paciente após correção ortodôntica (um mês de duração) e correção através de cirurgia gengival (um mês de cicatrização).

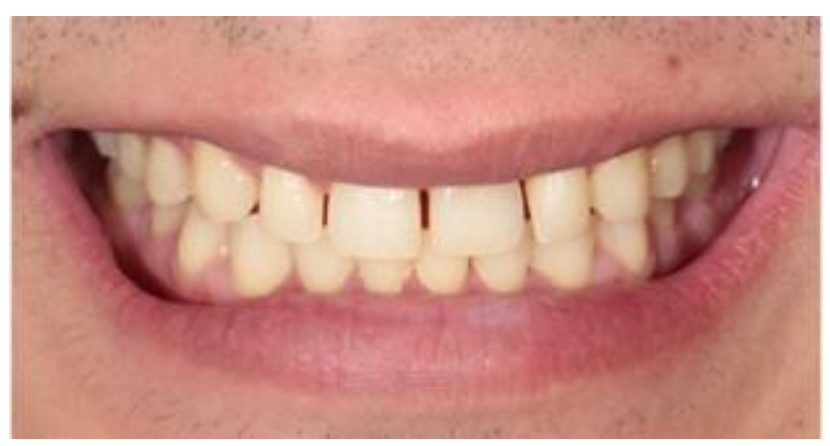

Figura 6: Aspecto intraoral após correção ortodôntica (um mês de duração) e correção através de cirurgia gengival (um mês de cicatrização).

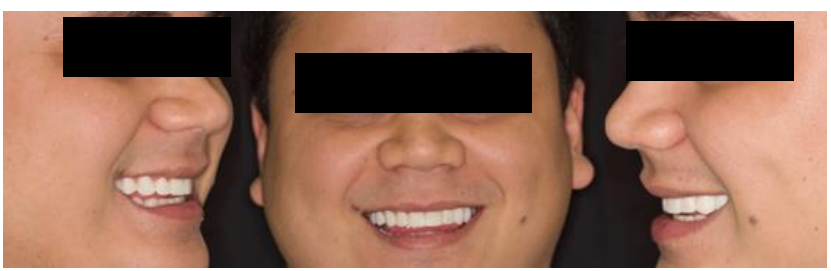

Figura 7: Simulação com mock-up.

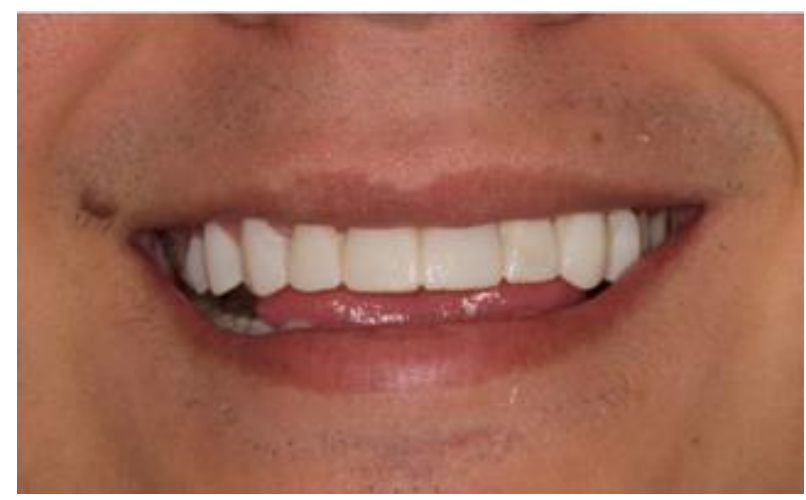

Figura 8: Mock-up: aspecto intraoral.

As cerâmicas foram testadas em 2 etapas: prova seca, sem nenhum cimento, somente testando a adaptação; prova com try-in, cimento teste, verificando assentamento e cor. Em seguida iniciou a cimentação definitiva utilizando o cimento resinoso Alcem Venner (FGM). Foi realizado o condicionamento das cerâmicas com ácido fluorídrico10\% por 20 segundos, em seguida lavado por 60 segundos, secado e realizada a aplicação do agente de união silano (ALGELUS) de acordo com a orientação do fabricante, posteriormente, o adesivo 
(UNIVERSAL 3M) foi aplicado. No dente foi realizada a hibridização com ácido fosfórico 32\% (FGM) e adesivo (UNIVERSAL 3M) por fim, o cimento foi adicionado na superfície interna das cerâmicas e levado em posição, tirados os excessos com fio dental, pincel ponta achatada e tiras de poliéster. Esse precedimento foi feito um a um e depois aplicado vaselina e polimerizado todas as lentes juntas por 40 segundos em cada face. Pequenos excessos remanescentes foram removidos com láminas de bisturi e tiras finas de lixa de aço extra-fina.

O controle foi realizado após 6 meses e também após 1 ano de tratamento (Figuras 9 à 16), sem apresentar alteração em suas estruturas,foi sondado e avaliado os terminos dos trabalhos e todos apresentavam boa adaptação e sem excessos. Apenas foi novamente ressaltado que o paciente deve melhorar o padrão de escovação por continuar apresentando biofilme e também gengivite.

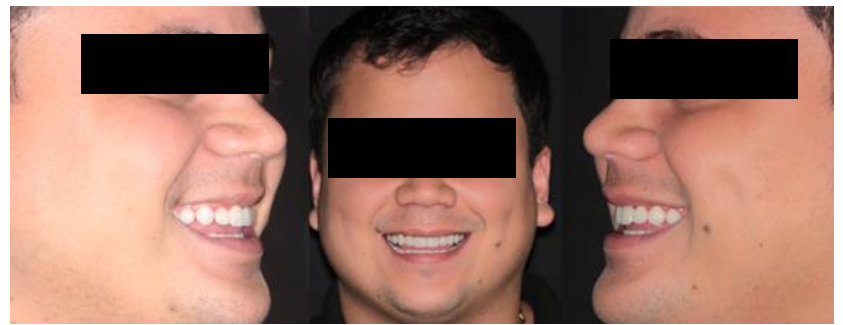

Figura 9: Pós-operatório após um ano de cimentação das facetas dentárias.

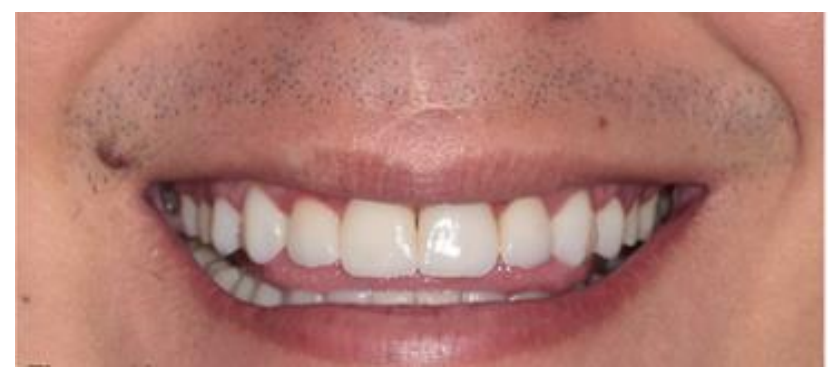

Figura 10: Pós-operatório após um ano de cimentação das facetas dentárias.

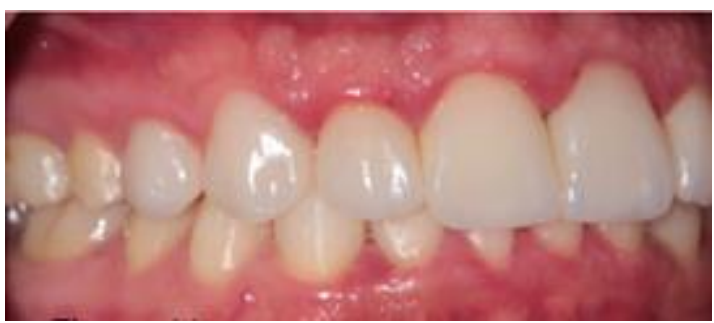

Figura 11: Pós-operatório após um ano de cimentação das facetas dentárias: vista intraoral lateral direita.

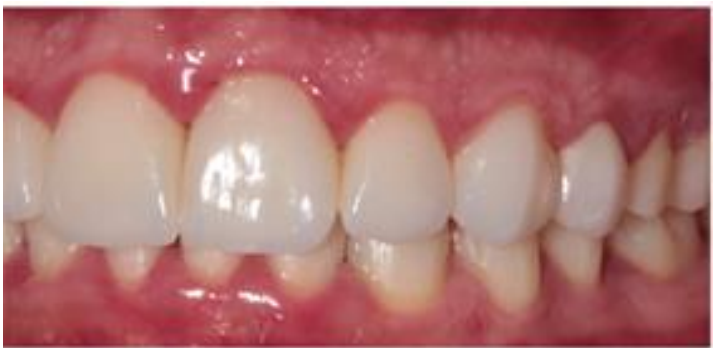

Figura 12: Pós-operatório após um ano de cimentação das facetas dentárias: vista intraoral lateral esquerda.

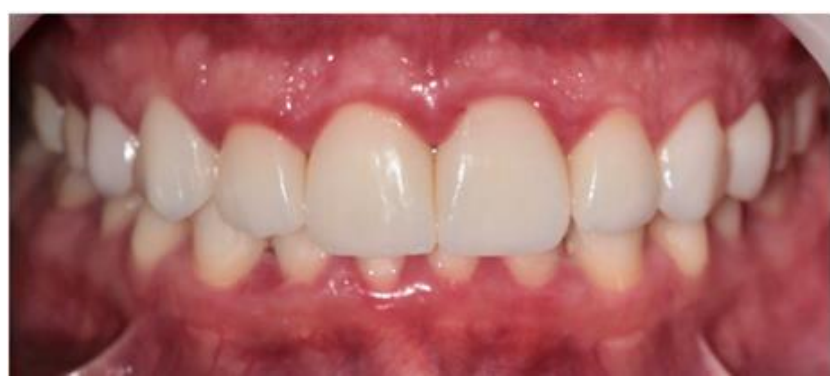

Figura 13: Pós-operatório após um ano de cimentação das facetas dentárias: aspecto intraoral frontal.

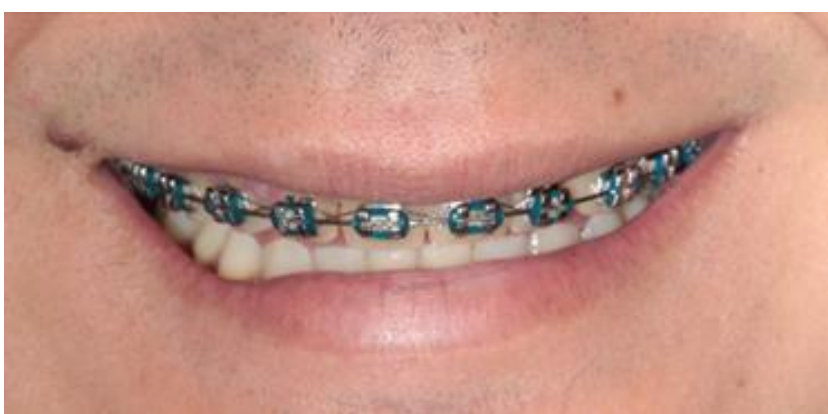

Figura 14: Aspecto intraoral frontal durante a correção ortodôntica.

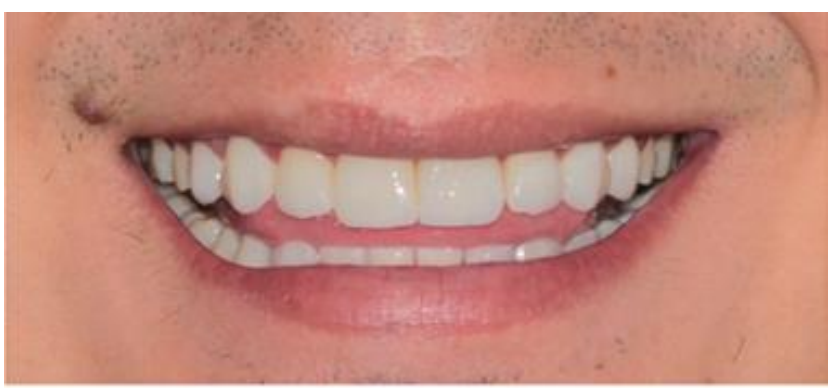

Figura 15: Aspecto intraoral frontal após tratamento ortodôntico e restaurador e fechamento dos diastemas.

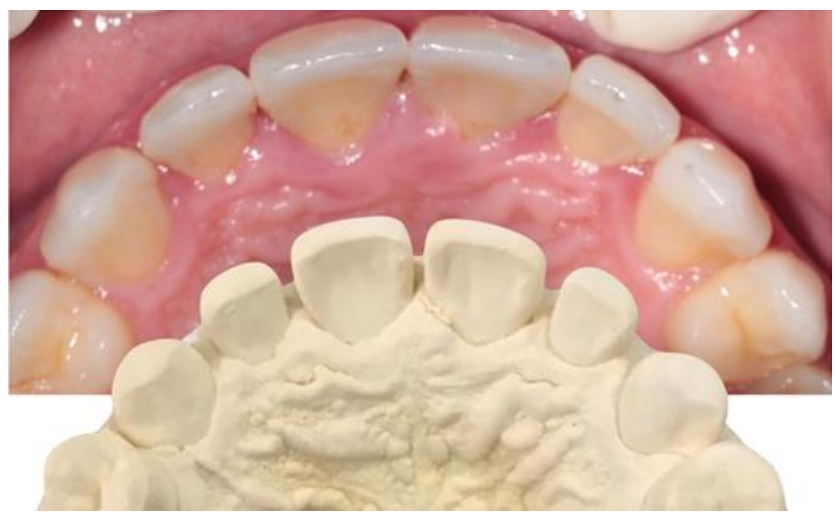

Figura 16: Imagem comparativa dos diastemas visíveis no modelo de gesso e a harmonia do arco facial vestibular encontrada com as cerâmicas.

\section{DISCUSSÃO}

Os diastemas dentários são espaços entre dois elementos dentários, que possuem aspecto de normalidade na infância. De um modo geral, o crescimento maxilar aliado à troca dos dentes decíduos pelos permanentes, possibilita o fechamento desse espaço. A manutenção dos espaços dentários possui etiologia multifatorial, geralmente relacionada à inserção do freio labial próxima à papila incisiva (freio labial hipertrófico), hereditariedade, presença de dentes intraósseos ou de hábitos deletérios ${ }^{4-6}$. 
$\begin{array}{cccc}\text { Pacientes } & \text { que possuem essa condição } \\ \text { anseiam por } & \text { um } & \text { sorriso } & \text { harmônico. }\end{array}$ Tradicionalmente, o tratamento de diastemas envolve o uso de terapia ortodôntica ou suporte, como no caso clínico descrito. No entanto, avanços na Odontologia cosmética apresentam ao paciente soluções restauradoras para o fechamento desses espaços com o incremento de materiais resinosos ou cerâmicos podendo ser facetas ou lentes de contato ${ }^{4,7}$.

No caso descrito optou-se por lâminados cerâmicos à base de dissilicato de lítio, pela mesma apresentar propriedades favoráveis, tais como: resistência à compressão, condutibilidade térmica, semelhança aos tecidos dentais, radiopacidade, integridade marginal, estabilidade de cor, biomimetismo, e por apresentar superioridade estética $^{8,9}$. Comparando-se ainda, facetas confeccionadas em resina composta e cerâmica, as últimas apresentam vantagens como estabilidade de cor por um período de tempo maior, alta resistência ao desgaste, maior resistência mecânica a fratura, proporcionando longevidade clinica ${ }^{1,10}$, além de que as restaurações de cerâmica oferecem um resultado previsível e bem sucedido, com uma probabilidade de sobrevida estimada em 93,5\% em 10 anos, sendo uma excelente escolha de tratamento ${ }^{11,12}$.

As cerâmicas à base de dissilicato de lítio (IPS E-max, IvoclarVivadent, Schaan, Liechtenstein) foram formuladas para diversas indicações: confecção de coroas de cerâmica pura, inlays, onlays, facetas, lentes de contato e confecção de próteses parciais fixas de três elementos, envolvendo dentes anteriores e posteriores ${ }^{13,14}$, porém, o sucesso de restaurações indiretas, depende do diagnóstico e planejamento corretos, desenho adequado dos preparos, bom desempenho profissional e amplo conhecimento clínico, além da escolha do agente cimentante conveniente e da técnica de cimentação correta $^{15,16}$.

Os agentes cimentantes devem preencher a interface entre o dente preparado (suporte) e a restauração, conferindo retenção, resistência à restauração e ao remanescente dentário, promovendo vedamento marginal e favorecendo a longevidade dos trabalhos protéticos. Portanto, um agente cimentante ideal deveria ter resistência mecânica e ser insolúvel aos fluidos orais ${ }^{15-17}$. Para este tipo de restauração o uso de cimentos exclusivamente foto-ativáveis, é fundamental para a manutenção da estética e estabilidade da cor, pois a pequena espessura destas restaurações não permite mascarar as alterações de cor de cimentos quimicamente ativados ${ }^{1,18,19}$. Esse material tem sido considerado de escolha nesse tipo de caso, pelo fato de que os cimentos quimicamente ativados e duais apresentam em sua composição amina terciária como ativador químico, que pode provocar alterações de cor com o passar do tempo, comprometendo o resultado estético ${ }^{1,20}$.
Desta forma, é necessário obter conhecimento dos aspectos técnicos inerentes ao tratamento proposto com as restaurações minimamente invasivas bem como a escolha de um sistema cerâmico adequado para obtenção dos resultados previstos ${ }^{21}$. Assim como, conhecer a forma, proporção, grau de aparecimento, cor, textura dos dentes e expectativa do paciente. Além disso, análise da estética vermelha, ou seja, da arquitetura gengival (altura do sorriso, zênites gengivais, perfil de emergência, etc.) que emoldura todos os dentes e sorriso é um aspecto fundamental na excelência do resultado final. Sendo assim, integrar as técnicas estéticas para a reabilitação dentária juntamente com cirurgias plásticas gengivais é extremamente necessário, como no caso apresentado, para se alcançar o resultado desejado ${ }^{22-25}$.

\section{CONSIDERAÇÕES FINAIS}

Neste caso clinico, as lentes de dissilicato de lítio, objetivavam o fechamento de diastemas presentes na região anterior, já que o uso de aparelho ortodôntico não foi suficiente para resolução do mesmo. Com a remoção do aparelho foi possível observar com a clareza o excesso de tecido gengival, na qual também era motivo de descontentamento em relação ao paciente, sendo então encaminhado para a remoção do mesmo. Lançou-se mão do mock-up, um ponto importante, pois por meio dele foi obtido um diagnóstico e um melhor planejamento do caso. Deuse então inicio aos preparos minimamente invasivos dos elementos 15 ao 25 . Outro ponto importante foi uso de cimento resinoso foto-ativado, demonstrandose de grande valia, já que o mesmo apresenta característica de estabilidade de cor superior comparado aos demais agente cimentantes.

Desta forma, é possível observar que cada etapa do tratamento proposto deve ser respeitada e rigorosamente realizada, sem negligências. Tendo em vista o correto diagnóstico e planejamento, levando ainda em consideração a opinião do paciente, seguindo tais passos teremos um resultado satisfatório para ambos os lados, tanto paciente quanto a equipe odontológica.

\section{REFERÊNCIAS}

1. Soares PV, Zeola LF, Souza PG, Pereira FA, Milito GA, Machado AC. Reabilitação Estética do Sorriso com Facetas Cerâmicas Reforçadas por Dissilicato de Lítio. Rev Odontol Bras Central. 2012;21(58):538-43.

2. Pena CE, Ortega LF, Soares RD, Coelho AS, Falchi F. A importância do planejamento multidisciplinar em casos de uso de cerâmicas com pouco ou nenhum desgaste em área estética: relato de caso clínico. J Clin Dent Res. 2017;14(1):60-7.

3. Angrisani Neto S, Pereira MA, Cunha LF, Garcia PP. Tratamentos estéticos conservadores para o 
fechamento de diastemas: resinas compostas e cerâmicas odontológicas. Rev Dental Press Estét. 2013;10(4):94-106.

4. Nishimori LE, Yokoyama AK, Marson FC, Silva CO, Corrêa GO. Lentes de contato: solução para diastemas anteriores. Rev Dental Press Estét. 2014;11(1):94-101.

5. Araújo LG, Bolognese MA. Diastema interincisal $\mathrm{x}$ freio labial anormal. Rev Bras Odontol. 1983;5:20-8.

6. Okida RC, Filho AJ, Barao VA, dos Santos DM, Goiato MC. The use of fragments of thin veneers as a restorative therapy for anterior teeth disharmony: a case report with 3 years of followup. J Contemp Dent Pract. 2012;13(3):416-20.

7. Kina S. Cerâmicas dentárias. Rev Dental Press Estét. 2005;2(2):111-28.

8. Amoroso AP, Ferreira MB, Torcato LB, Pellizzer EP, Mazaro JVQ, Filho GH. Cerâmicas odontológicas: propriedades, indicações e considerações clínicas. Rev Odontol Araçatuba. 2012;33(2):19-25.

9. Savaris DI, Vermudt A, Ghizoni JS, Pamato S, Pereira JR. Lentes de contato harmonização e estética com preparos conservadores. J Res Dent. 2018;6(4):91-7.

10. Cardoso PC, Cardoso LC, Decurcio RA, Monteiro LJE. Restabelecimento Estético Funcional com Laminados Cerâmicos. Rev Odontol Bras Central 2011;20(52):88-93.

11. Moraes AB, Webber MBF, Marson FC, Progiante PS, Silva CO, Lolli LF. Reanatomização estética multidisciplinar em dente lateral conoide. Rev Dental Press Estét. 2013;10(4):50-7.

12. Mehotra KK, Saimbi CS, Jha T. Microdontic maxillary lateral incisor: case report. J Clin Ped Dent. 1992;16(2):119-28.

13. Mazioli CG, Peçanha MM, Daroz LGD, Siqueira CA, Fraga MAA. Resistência de união de diferentes cimentos resinosos a cerâmica à base de dissilicato de lítio. Rev Odontol UNESP. 2017; 46(3):174-78.

14.Fabianelli A, Goracci C, Bertelli E, Davidson CL, Ferrari M. A clinical trial of Empress II porcelain inlays luted to vital teeth with a dual-curing adhesive system and a self- curing resin cement. J Adhes Dent. 2006;8(6):427-31.

15.Lopes MWF, Farias ABL, Cabral BLAL, Guerra CMF. Cimentação em prótese: procedimentos convencionais e adesivos. Int J Dent. 2007; 6(2):58-62.

16. Figueiredo AR, Castro Filho AA, Matuda FS. Cimentação provisória e definitiva. In: Cardoso RJA, Gonçalves EAN (COORD). Oclusão/ATM, Prótese, Prótese sobre implantes e Prótese Bucomaxilofacial. São Paulo: Artes Médicas; 2002. cap. 15.
17.Bottino MA. Estética em Reabilitação Oral Metal Free. São Paulo: Artes Médicas; 2001.

18. Sensi L, Baratieri LN, Monteiro Junior S. Cimentos Resinosos. In:Kina S, Brugrera A. Invisível: Restaurações estéticas cerâmicas. Maringá: Dental Press; 2007. p. 303-19. 36.

19.Karaagaclioglu L, Yilmaz B. Influence of cement shade andwater storage on the final color of leucite-reinforced ceramics. Oper Dent. 2008;22(4):286-91.

20. Aquino APT, Cardoso PC, Rodrigues MB, Takano AE, Porfírio W. Facetas de Porcelana: Solução Estética e Funcional. International J Braz Dent. 2009;5(2):142-52.

21.Zavanelli AC, Zavanelli RA, Quinellimazaro JV, de Paula WN, Borges MA, Bagio DM. Associação de preparos minimamente invasivos e plástica gengival: relato de caso clínico. Arch Health Invest. 2015;4(3):1-9.

22. Sene F. Plástica gengival e microlaminados cerâmicos para reabilitação estética do sorriso. Rev Dental Press Estét. 2015;12(4):79-90.

23.Wang $X$, Fan D, Swain MV, Zhao K. A systematic review of all-ceramic crowns: clinical fracture rates in relation to restored tooth type. Int J Prosthodont. 2012;25(5):441-50.

24.Layton DM, Clarke M. A systematic review and metaanalysis of the survival of non-feldspathic porcelain veneers over 5 and 10 years. Int $\mathrm{J}$ Prosthodont. 2013;26(2):111-24.

25. Lee YK, Cha HS, Yu B. Illuminating lightdependent color shifts in core and veneer layers of dental allceramics. J Biomed Opt. 2014;19(9):95002.

\section{CONFLITO DE INTERESSES}

Os autores declaram não haver conflitos de interesse.

\section{AUTOR PARA CORRESPONDENCIA}

\section{Lia Beatrice}

lia_beatrice@ hotmail.com

Submetido em 29/10/2018

Aceito em 12/03/2019 